\title{
Parallel kinetic resolution of racemic ketones through reduction: a quantitative analysis
}

\author{
Juan R. Dehli and Vicente Gotor* \\ Departamento de Química Orgánica e Inorgánica, Universidad de Oviedo, \\ c/ Julián Clavería 8, 33071 Oviedo, Spain \\ E-mail:vgs@sauron.quimica.uniovi.es
}

\begin{abstract}
Dedicated to Professor Marcial Moreno-Mañas on the occasion of his $60^{\text {th }}$ birthday (received 12 Dec 01; accepted 26 May 02; published on the web 03 Jun 02)
\end{abstract}

\begin{abstract}
Equations and useful graphs for the quantitative treatment of ketone reductions through a parallel kinetic resolution have been developed. They can be applied to a hypothetical iterative process, so that a prediction of its viability can be done.
\end{abstract}

Keywords: Parallel kinetic resolution, ketones, alcohols, asymmetric synthesis

\section{Introduction}

In the last years, the preparation of enantiopure compounds has become an expanding area of the fine chemical industry, due to the general awareness of the physiological and ecological advantages of the use of single enantiomers. ${ }^{1}$ With this purpose, several chemical and biochemical strategies have been developed to either prepare them from prochiral compounds (asymmetrization) or from racemic mixtures (resolution).

In a classical kinetic resolution (CKR), ${ }^{2}$ one of the enantiomers of a racemic mixture is converted much faster than the other one, so that (ideally) at $50 \%$ conversion, one can obtain both the product and the substrate in enantiomerically pure form. However, this is not always the case, and special care has to be taken in order to stop the reaction at appropriate conversion values, so that the product or the substrate can be obtained in satisfactory ee. This is due to the build-up of the less reactive enantiomer, and therefore its relative rate.

To overcome this problem, this less reactive enantiomer can be removed in a parallel reaction, ideally in a similar rate. This new strategy has been termed parallel kinetic resolution (PKR). ${ }^{3}$ To date, a few chemical ${ }^{4}$ and enzymatic ${ }^{5}$ examples have been described. But all of them suffer from the impossibility of recovering the enantioenriched substrate.

Within our ongoing interest in the synthesis of optically active amino alcohols through enzymatic methods, ${ }^{6}$ we have recently described our results on the biotransformations of $\alpha$-non substituted $\beta$-keto nitriles by whole cells. ${ }^{7}$ Then, we decided to study the bioreduction of $\alpha$-fully 
substituted $\beta$-keto nitriles. ${ }^{8}$ Due to the existence of a chiral centre in the substrate, the reduction of the carbonyl group in both enantiomers of the substrate following Prelog's rule ${ }^{9}$ yielded a mixture of enantioenriched diastereomers, easily separated by chromatography (see scheme 1).

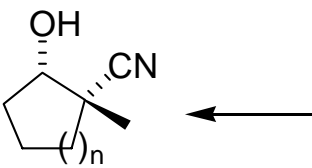

I-2<smiles>CC1(C#N)[Te]CCC1=O</smiles>

(S)-1<smiles>C[C@@]1(C#N)[CH]CCC1=O</smiles>

(R)-1

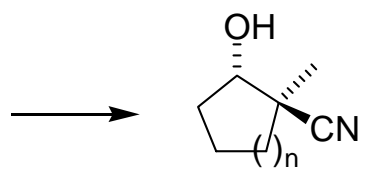

$u-2$

\section{Scheme 1}

To the best of our knowledge, this constitutes the first example of a PKR of a racemic ketone via reduction. Furthermore, the ketone can be recovered in optically active form. In this paper, we present some useful equations that relate the different variables involved in the process.

\section{Results and Discussion}

In the process (see eq. 1 and 2), both enantiomers of the ketone ( $R$ and $S$ ) are transformed in up to four stereoisomers of the corresponding secondary alcohol (RR, $R S, S R$ and $S S$ ).

$$
\begin{aligned}
& R \rightarrow R R+R S \\
& S \rightarrow S R+S S
\end{aligned}
$$

In such a process, a double stereoselectivity can be considered: on one hand, a substrate enantiodiscrimination can occur; the reducing agent reacts much faster with one of the enantiomers (CKR). The other limit situation is a (high) diastereoselectivity for one of the prochiral faces of the carbonyl group, independently of the configuration of the substrate (PKR). In this paper we will focus on this second situation.

For clarity, we will assume the following hypotheses:

a) the priority order of the substituents does not change during the reaction.

b) the reduction takes place following Prelog's rule (i.e., creating a new chiral center of $S$ absolute configuration), therefore yielding $R S$ and $S S$ as the major products.

For every enantiomer of the substrate, the diastereoselectivity of the reduction is expressed by the epimeric excess (ep) of the products, defined as:

$$
\begin{aligned}
& e p_{R}=\frac{R S-R R}{R S+R R} \times 100 \\
& e p_{S}=\frac{S S-S R}{S S+S R} \times 100
\end{aligned}
$$


And for every diastereomer of the product ( $u$ and $l$ ) obtained in the global reduction, the stereoselectivity is expressed by their enantiomeric excess $(e e)$ :

$$
\begin{aligned}
& e e_{u}=\frac{R S-S R}{R S+S R} \times 100 \\
& e e_{l}=\frac{S S-R R}{S S+R R} \times 100
\end{aligned}
$$

\section{1.- Reduction of a racemic ketone}

In the particular case that we use a racemic ketone as substrate, the initial concentration of both enantiomers $\left(R_{o}\right.$ and $\left.S_{o}\right)$ is identical. Therefore, after complete conversion, the mass balance of the process holds:

$$
R_{o}=R R+R S=S R+S S=S_{o}
$$

From eq. (3) and (7), one gets:

$$
R S=\frac{100+e p_{R}}{200} R_{o}
$$

and from eq. (4) and (7):

$$
S R=\frac{100-e p_{S}}{200} S_{o}
$$

Introducing eq. 8 and 9 in eq. 5:

$$
e e_{u}=\frac{e p_{R}+e p_{S}}{200+e p_{R}-e p_{S}} \times 100
$$

In a similar way, an equivalent expression for $e e_{l}$ can be obtained:

$$
e e_{l}=\frac{e p_{R}+e p_{S}}{200+e p_{S}-e p_{R}} \times 100
$$

The third stereochemical characteristic of the system at the end of the reaction is the diastereomeric excess $(\mathrm{de}) \mathrm{:}^{10}$

$$
d e=\frac{l-u}{l+u} \times 100=\frac{(S S+R R)-(S R+R S)}{S S+R R+S R+R S} \times 100
$$

From eq. (3), (4) and (7), it can be transformed into:

$$
d e=\frac{e p_{S}-e p_{R}}{2}
$$

This variable is the less valuable one: in fact, in the ideal case of perfect stereoselectivity of the $\mathrm{PKR}, e p_{S}=e p_{R}=100$ and, therefore, $d e=0$.

Figure 1 shows graphical representations of $e e_{u}$ and $e e_{l}$ vs. $e p_{R}$ and $e p_{S}$. From them it is clear that $e e_{u}$ shows a much stronger dependence on $e p_{S}$ than on $e p_{R}$. As might be expected, the opposite happens to $e e_{l}$. That is to say, the ee of a diastereomer is mainly dependent on the 
diastereoselectivity of the reduction of the enantiomer of the ketone that yields the other diastereomer.

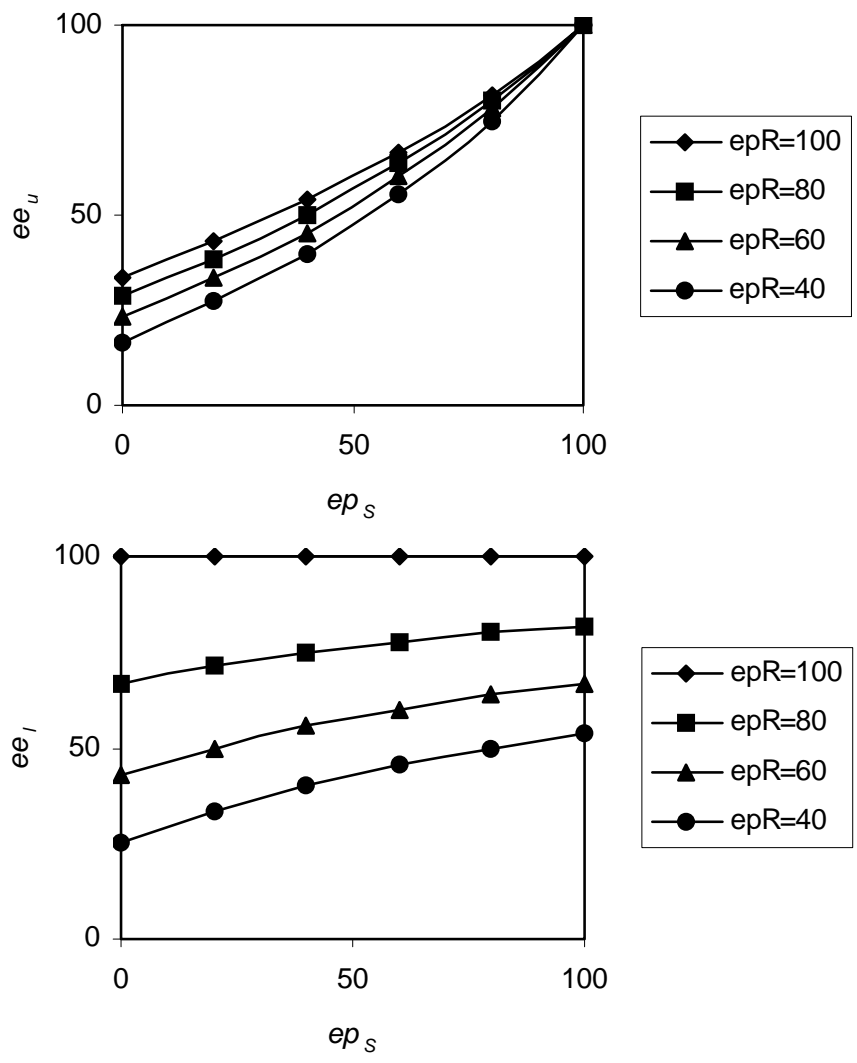

Figure 1. Computer generated plot of $e e_{l}$ and $e e_{u}$ as functions of $e p_{R}$ and $e p_{s}$.

\section{Iterative reduction-oxidation of a ketone}

An interesting feature of the PKR of ketones via reduction, is that the substrate can be recovered in an optically active form by oxidation of the alcohol. Therefore, the process can be repeated if the ee values obtained are not satisfactory yet.

Now, let us assume that the ketone subjected to reduction has an initial enantiomeric excess $\left(e e_{k}\right):{ }^{11}$

$$
e e_{k}=\frac{S_{o}-R_{o}}{S_{o}+R_{o}} \times 100=\frac{S S+S R-R S-R R}{S_{o}+R_{o}} \times 100
$$

The chiral balance ${ }^{12}$ of the process holds:

$$
\begin{aligned}
& R_{o}=R S+R R \\
& S_{o}=S S+S R
\end{aligned}
$$

From eq. (14), (15) and (16), it can be obtained:

$$
R S+R R=\frac{R_{o}+S_{o}}{2}\left(1-\frac{e e_{k}}{100}\right)
$$

which, using eq. (3), can be converted to:

$$
R S=\frac{\left(100+e p_{R}\right)\left(100-e e_{k}\right)\left(R_{o}+S_{o}\right)}{40000}
$$


Similar expressions can be obtained for $R R$, $S S$ and $S R$. These, introduced in eq. 5 and 6 , give the following general expressions for $e e_{u}$ and $e e_{l}$ :

$$
\begin{aligned}
& e e_{u}=\frac{e p_{R}+e p_{S}-\frac{e e_{k}}{100}\left(200+e p_{R}-e p_{S}\right)}{200+e p_{R}-e p_{S}-\frac{e e_{k}}{100}\left(e p_{R}+e p_{S}\right)} \times 100 \\
& e e_{l}=\frac{e p_{R}+e p_{S}+\frac{e e_{k}}{100}\left(200+e p_{S}-e p_{R}\right)}{200+e p_{S}-e p_{R}+\frac{e e_{k}}{100}\left(e p_{R}+e p_{S}\right)} \times 100
\end{aligned}
$$

And for de, a general expression from eq. 3, 4, 15 and 16 can be developed:

$$
d e=\frac{100+e e_{k}}{200} e p_{s}-\frac{100-e e_{k}}{200} e p_{R}
$$

Obviously, eq. 19, 20 and 21 reduce to eq. 10, 11 and 12 respectively for the particular case in which a racemic ketone is used as substrate $\left(e e_{k}=0\right)$.

Since $e e_{k}$ is actually the $e e$ of the alcohol obtained in the previous reduction (assuming no racemization takes place under the oxidation conditions), wether an iterative reduction-oxidation process is worth it can be predicted a priori. That is to say, the diastereoselectivity of the reduction of both enantiomers of the starting racemic ketone (quantified by $e p_{R}$ and $e p_{S}$ ) determines not only the ee of the diastereomers of a "first" reduction, but also the numbers of cycles which are necessary to reach a desired ee.

Figure 2 represents the ee of the alcohol as a function of the diastereoselectivity of the reduction and the number of cyles. In the first case, we have considered a process in which both enantiomers of the ketone are reduced with the same diastereoselectivity $\left(e p_{R}=e p_{S}=e p\right)$. Of course, in this case, the $e e$ of both diastereomers is the same $\left(e e_{u}=e e_{l}=e e\right)$. It is, in fact, the ideal case mentioned above: the reduction of the prochiral carbonyl is independent of the rest of the molecule. It is worth mentioning that this cyclic strategy allows to obtain ee as high as needed, even if both ep values are relatively low.

The second case is the one we have observed in the bioreduction of $\beta$-keto nitriles: one of the enantiomers is reduced with complete diastereoselectivity $\left(e p_{S}=100\right)$, while the other is not. In this case, the $u$ isomer is always obtained enantiopure, while the $e e_{l}$ can be increased by iterating the process. 
a)

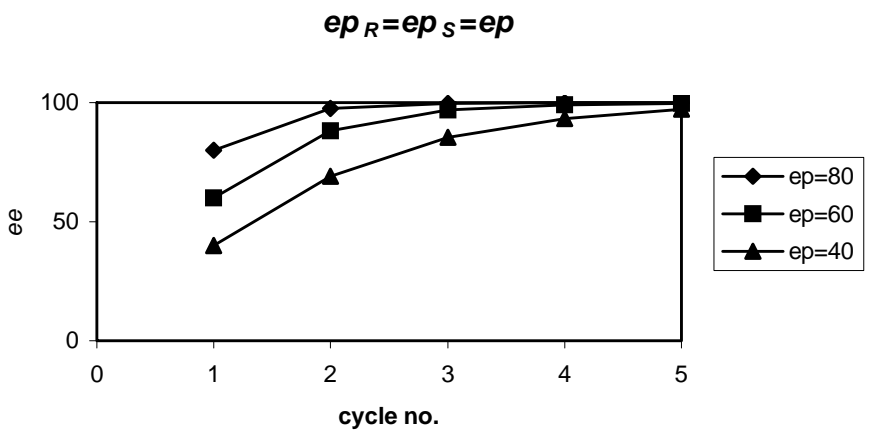

b)

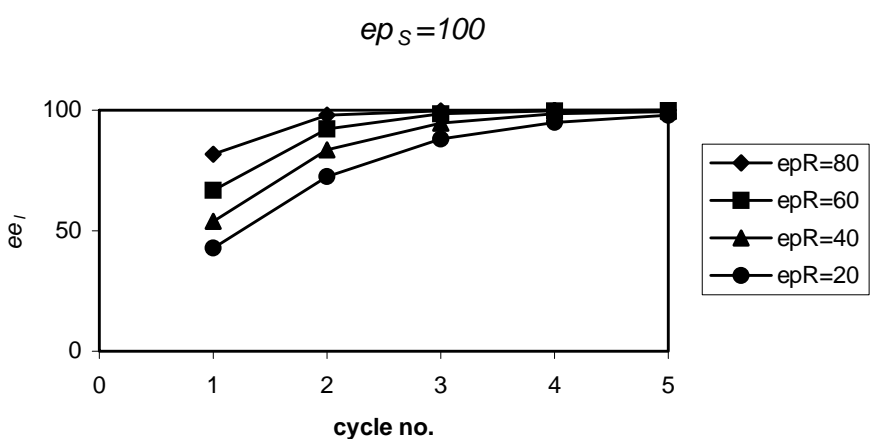

Figure 2. Cyclic enantioselective reduction - non enantioselective oxidation of ketones.

Dependence of the ee of the alcohol on ep and cycle number, when: a) both ep are the same; b) one of the ep $\left(e p_{S}\right)$ is 100 .

Although both graphics are qualitatively very similar, there is one big difference between both situations, as far as the application of this strategy in a synthetic route is concerned: in the last one, $u$ is always obtained enantiopure, independently of the initial $e e_{k}$. Thus, it is only necessary to oxidize the $l$ to give the $S$ ketone, which is then subjected to a second reduction. Therefore, in every cycle, we accumulate $u$ up to $50 \%$ chemical yield and the $l$ diastereomer is obtained in higher ee.

However, in the other limit situation $\left(e p_{R}=e p_{S}\right)$, we do not obtain any diastereomer enantiopure after the first reduction. It is necessary to oxidize both, and reduce them separately. And in this second reduction, together with a major proportion of the diastereomers with a higher $e e$, we will obtain a minor proportion of racemic diastereomers: for instance, if we take the $l$ alcohol, and oxidize it, we will get the $S$ ketone with the same $e e_{l}$. A second reduction will yield $l$ in higher $e e$, but also a smaller quantity of racemic $u$ whose quantity will depend on the $e e_{k}$. Therefore, for practical use, ep must be high to assure not only a low number of cycles, but also a big quantity of the alcohol with higher ee (which can be deduced from eq. 21).

The latter represents one of the limitations of this methodology: at least one of the ep must be high enough, so that high ee and chemical yields can be obtained in a few cycles. Another limitation is the separation of the diastereomers: if they are not efficiently separated, (partial) racemization will take place. And concerning the validity of this mathematical approach, the stereoselectivity of the reduction must be independent of the substrate concentration ( $e e_{p}$ must be constant). 
In summary, the relationship between the different stereochemical variables involved in a reduction of a racemic ketone through PKR has been described. This strategy can be repeated to obtain higher ee values, if needed. This cyclic strategy has been also studied, so that a prediction of the viability of the process can be done. Further work is currently in progress in our laboratory, concerning the application of this methodology to the preparation of enantiopure ketones and alcohols.

\section{Acknowledgements}

Financial support of this work by the Spanish Ministerio de Ciencia y Tecnología (project BIO 98-0770) is greatfully acknowledged. J.R.D. thanks the Spanish Ministerio de Educación, Cultura y Deporte for a predoctoral fellowship.

\section{References}

1. Sheldon, R. A. Chirotechnology. Industrial Synthesis of Optically Active Compounds. J. Wiley: New York, 1994.

2. (a) Kagan, H. B.; Fiaud, J. C. Top. Stereochem. 1988, 18, 249. (b) Sih, C. J.; Wu, S. H. Top. Stereochem. 1989, 19, 63.

3. Eames, J. Angew. Chem., Int. Ed. 2000, 39, 885.

4. (a) Doyle, M. P.; Dyatkin, A. B.; Kalinin, A. V.; Ruppar, D. A.; Martin, S. F.; Spaller, M. R.; Liras, S. J. Am. Chem. Soc. 1995, 117, 11021; (b) Vedejs, E.; Chen, X. J. Am. Chem. Soc. 1997, 119, 2584; (c) Bertozzi, F.; Crotti, P.; Macchia, F.; Pineschi, M.; Feringa, B. L. Angew. Chem., Int. Ed. 2001, 40, 930 (d) Pedersen, T. M.;. Hansen, E. L.; Kane, J.; Rein, T.; Helquist, P.; Norrby, P.O.; Tanner, D. J. Am. Chem. Soc. 2001, 123, 9738.

5. (a) Alphand, V.; Furstoss, R. J. Org. Chem. 1992, 57, 1306; (b) Adger, B.; Teresa Bes, M.; Grogan, G.; McCague, R.; Pedragosa-Moreau, S.; Roberts, S. M.; Villa, R.; Wan, P. W. H.; Willets, A. J. J. Chem. Soc., Chem. Commun. 1995, 1563.

6. (a) García-Urdiales, E.; Rebolledo, F.; Gotor, V. Tetrahedron: Asymmetry 1999, 10, 721; (b) Luna, A.; Maestro, A.; Astorga, C.; Gotor, V. Tetrahedron: Asymmetry 1999, 10, 1969; (c) Gotor, V. Bioorg. Med. Chem. 1999, 10, 2189.

7. (a) Gotor, V.; Dehli, J. R.; Rebolledo, F. J. Chem. Soc., Perkin Trans. 1 2000, 307; (b) Dehli, J. R.; Gotor, V. Tetrahedron: Asymmetry 2000, 11, 3693; (c) Dehli, J. R.; Gotor, V. Tetrahedron:Asymmetry 2001, 12, 1485.

8. Dehli, J. R.; Gotor, V. J.Org. Chem, 2002, 67, 1716.

9. Prelog, V. Pure Appl. Chem. 1964, 9, 119.

10. It has been assumed that the major diastereomer is $l$. If the de obtained has a negative value, it means that the $u$ is obtained as the major one.

11. In our following equations, we will assume that the ketone in enriched in the $S$ antipode. If this were not the case, one should introduce a negative value for the $e e_{k}$.

12. Straathof, A. J. J.; Rakels, J. L. L.; Heijnen, J. J. Biotechnol. Bioeng. 1995, 45, 536. 